\title{
APPENDIX
}

\section{Cast, Music and Production for Passion, Lament, Glory}

\author{
Performed St Paul's Cathedral Melbourne, 31 March and 1 April 2017
}

\section{Cast}

Salve Regina: Jacqueline Porter (Soprano Soloist)

Narrators: Andreas Loewe (John) and Heather Fletcher (Woman)

Jesus: Tim Rutty

Soldiers: Nathan Wright, Alastair Cooper-Golec

Mary: Jordan Auld

Stabat Mater performers: Ruth Blythman, Stella Joseph-Jarecki, Elspeth Bawden, Olivia O’Brien, Amelia Wawarzon, Kristina Fekonja, Emilia Bertolini, Olivia Federov-Yemm, Sarah Louise Amos, Leah Philips, Millie Leaver, Hannah Kostros and Heather Fletcher

The Messiah Chorus: Patrick Anderson, Molly Athanatos, Jade Biezen, Caitlyn Bosch, Lisette Botton, Tatjana Brandson, Saffrey Brown, Francesca Browne, Isabela Calderon, Tessa Canning, Danielle Carey, Dylan Casey, Paul Chantler, Jessica Cleave, Stephen Coutts, Rhiannon Curtis, Nicola Czaplinski, Michael Dimovski, Jessie Eastwood, Brianna Ekberg, James Emerson, Joshua Erdelyi-Getz, Rebecca Fullerton, Grace Gallur, Ben Glover, Ivana Gonzales, Esther Gresswell, Sofia Habel, Alexandra Hadji, Emma Hamley, Carmen Hammelmann, Amanda Hargreaves, Samantha Hargreaves, Chloe Harris, Tom Harvey, Ryan Hayward, Sarah Hollibone, Lane Hyde, Teresa Ingrilli, Olivia Jackson, Shabrika Jeyakody, Shajeda Kalitzki, Bridgette Kelsey, Emily Laird, Aurora Lee, Xiaonan Liang, Dorcas Lim, Jacob Lingard, Lana Lowry, Patrick MacDevitt, Coco Martel, Aidan McGartland, Grace McGuigan, Phillipa McQuinn, Rebecca Mitchell, Stephanie Morgan, Matilda Morley, Molly Murnane, Hannah Murphy, Jessica Nelson, India Ney, Selena Nicastri, Melanie O'Brien, Mairead O'Connor, Eloise O'Donnell, Albert O'Loughlin, Michael Padgett, Marianna Paradowski, Sarah Parkin, Beth Potter, Rory Preece, Vetalia Pribadi, Jennifer Ratcliffe, Samantha Robertson, Andre Sasalu, Emma-Mae Schmidt, Lauren Sibree, Ruby Smith, Cassandra Sulc, Madeleine Sullivan, Shimona evathasan, Chloe Toh, Caitlin Toohey, Syrah Torii, Billie Tumarkin, Imara Waldhart, Renee White, Eloise Whitehead, Angus Wilkinson, Greta Wilkinson, Simon Wright, Laura Zalesiak. 
Davidson, J. (2017). "Passion, Lament, Glory”: Baroque Music and Modern Social Justice Resonances. Voices: A World Forum For Music Therapy, 17(3). doi:10.15845/voices.v17i3.935

\section{Music Team}

Musical Director for Salve Regina and Stabat Mater: Erin Helyard

Conductor for Messiah excerpts: Stephen Grant

Repetiteur for the project: David Barnard

Violins, David Irving and Catherine Shugg; Viola, Christian Read; and Cello, Natasha Kraemer

\section{Production Team}

Artistic Director: Jane Davidson

Designer (Stage and Lighting): Matthew Adey

Production Manager: Paul Doyle

Production Assistant: Ruth Brightman

Costume Realisation: Matilda Woodroffe

Lighting Assistants: Michelle Thorne and Nick Glenn,

Rigging: Geoffrey Dunstan 